\title{
Prevalencia de ectoparásitos en cuyes (Cavia porcellus) de crianza familiar-comercial en el distrito de Matahuasi, Junín (Perú)
}

\section{Prevalence of ectoparasites in guinea pigs (Cavia porcellus) of family-commercial breeding in the district of Matahuasi, Junín (Peru)}

\author{
Fiorela Santos R. ${ }^{1}$, Rosa Pinedo V. ${ }^{1}$, Amanda Chávez V. ${ }^{1,2}$
}

\section{Resumen}

El objetivo del estudio fue determinar la prevalencia de ectoparásitos en cuyes (Cavia porcellus) de crianza familiar-comercial en el distrito de Matahuasi, Junín, Perú, así como identificar las especies parasitarias, tipo de asociación parasitaria y evaluar la asociación entre la presencia de ectoparásitos y las variables etapa productiva y sexo. Se evaluaron 299 cuyes entre enero y marzo de 2017. Los ectoparásitos fueron recolectados mediante cuatro técnicas (raspado profundo de piel, cinta adhesiva, tricograma y peinado fino). Los ectoparásitos fueron examinados por observación directa al microscopio a $10 \mathrm{X}$ y $40 \mathrm{X}$, y en algunos casos fueron aclarados con $\mathrm{KOH}$ al 10\%. Se encontró una prevalencia de $67.0 \pm 5.3 \%$, identificándose tres especies de acariformes $(63.0 \pm 5.5 \%) \mathrm{y}$ una especie de Phthiraptera $(12.0 \pm 3.7 \%)$. Dentro de los acariformes se identificó Ornithonyssus bursa (53\%), Chirodiscoides caviae (15\%) y Dermanyssus gallinae (7\%). La especie Phthiraptera fue Gliricola porcelli (12\%). El monoparasitismo fue el más frecuente $(75 \%)$. No se encontró asociación significativa entre la presencia de ectoparásitos y las variables etapa productiva y sexo.

Palabras clave: cuy, ácaros, piojos, crianza familiar-comercial

\section{Abstract}

The aim of this study was to determine the prevalence of ectoparasites in guinea pigs (Cavia porcellus) of family-commercial breeding in the district of Matahuasi, Junín, Peru, as well as to identify parasitic species, type of parasitic association and evaluate the association between the presence of ectoparasites and the productive stage and sex

\footnotetext{
${ }^{1}$ Laboratorio de Microbiología y Parasitología, Facultad de Medicina Veterinaria, Universidad Nacional Mayor de San Marcos, Lima, Perú

${ }^{2}$ E-mail: achavezv@unmsm.edu.pe
}

Recibido: 24 de mayo de 2019

Aceptado para publicación: 8 de abril de 2020

Publicado: 11 de agosto de 2020 
variables. A total of 299 guinea pigs were evaluated between January and March 2017. Ectoparasites were collected using four techniques (deep skin scraping, adhesive tape, trichogram and fine combing). The ectoparasites were examined by direct observation under the microscope at 10X and 40X, and in some cases they were rinsed with $10 \%$ $\mathrm{KOH}$. The prevalence of ectoparasites was $67.0 \pm 5.3 \%$, identifying three species of acariforms $(63.0 \pm 5.5 \%)$ and one species of Phthiraptera $(12.0 \pm 3.7 \%)$. Among the acariforms, Ornithonyssus bursa (53\%), Chirodiscoides caviae (15\%) and Dermanyssus gallinae (7\%) were identified. The Phthiraptera species was Gliricola porcelli $(12 \%)$. Monoparasitism was the most frequent (75\%). No significant association was found between the presence of ectoparasites and the productive stage and sex variables.

Key words: guinea pig, mites, lice, family-commercial breeding

\section{INTRODUCCIÓN}

El cuy (Cavia porcellus), mamífero roedor herbívoro, es básicamente utilizado como animal de producción en los países andinos, donde los problemas de piel forman parte de las complicaciones sanitarias, especialmente por ectoparásitos que, además, predisponen a infecciones secundarias (Chauca, 1997; Florián, 1999).

Los ectoparásitos son un factor importante dentro de las enfermedades parasitarias. Existen tres grupos importantes de ectoparásitos (piojos, pulgas y ácaros), los cuales tienen gran importancia epidemiológica, porque actúan como transmisores de microorganismos patógenos, como virus, bacterias, rickettsias, protozoarios y helmintos (Valim et al., 2004). Dittmar (2001) trabajando con 17421 cuyes (Cavia porcellus) en 14 departamentos del Perú encontró mayoritariamente pulgas como Pulex spp, Tiamastus cavicola, Xenopsylla cheopis, Ctenocephalides felis y Echidnophaga gallinacea, además de piojos como Gliricola porcelli, Trimenopon hispidum y Gyropus ovalis, y ácaros como Ornithonyssus bacoti, Dermanyssus gallinae y Chirodiscoides caviae.
En ceja de selva, Robles et al. (2014) determinaron la frecuencia de la parasitosis externa en cuyes de crianza familiar comercial, determinando frecuencias de 71 y $83 \%$ en las épocas de lluvia y seca, respectivamente, encontrando principalmente Chirodiscoides caviae y Gliricola porcelli, pero también Trixacarus caviae, Dermanyssus gallinae, Ornithonyssus spp, Cheyletiella spp, Trimenopon hispidum y Gyropus ovalis. Por otro lado, Gordillo (2015) encontró una prevalencia de $75.5 \%$ de ectoparásitos en 200 cuyes en la provincia de Arequipa, con mayor presencia en hembras (59.6\%). En ese estudio, se halló dos especies de ectoparásitos: Dermanyssus gallinae (30.5\%) y Echidnophaga gallinacea (20\%), además de biparasitismo en un $25 \%$.

Matahuasi, distrito del departamento de Junín, Perú, presenta una crianza de cuyes en pleno crecimiento, donde diversas asociaciones de criadores tienen asesoramiento técnico de entidades particulares. No obstante, muchos productores no manejan un adecuado manejo y control sanitario. El objetivo de este estudio fue estimar la prevalencia de ectoparásitos en cuyes (Cavia porcellus) de crianza familiar comercial en el distrito de Matahuasi, así como identificar las especies parasitarias, tipo parasitario (monoparasitismo, 
biparasitismo y poliparasitismo) y evaluar la asociación entre la ectoparasitosis y las variables etapa productiva y sexo.

\section{Materiales y Métodos}

\section{Lugar del Estudio}

El estudio se realizó en granjas de cuyes familiar-comercial del distrito de Matahuasi, provincia de Concepción (Junín, Perú), durante los meses de enero a marzo de 2017. El área de estudio está ubicada a $3279 \mathrm{msnm}$ con temperatura promedio anual de $10.8^{\circ} \mathrm{C}$. El procesamiento de muestras e identificación de ectoparásitos se llevó a cabo en el Laboratorio de Parasitología de la Facultad de Medicina Veterinaria de la Universidad Nacional Mayor de San Marcos, Lima.

\section{Tamaño Muestral}

El tamaño muestral fue determinado mediante la fórmula para evaluar una proporción basada en la aproximación normal a la distribución binomial (Daniel, 2007), con $95 \%$ de confianza y $5 \%$ de precisión. Se utilizó como prevalencia referencial $75.5 \%$ (Gordillo, 2015), obteniéndose un mínimo de 284 cuyes. No obstante, se trabajó con 299 animales criados en pozas, de 23 productores (13 animales/productor) distribuidos en cuatro zonas (Chimpamarca, Hualianta, Centro y 2 de Mayo) del distrito de Matahuasi. El $26 \%$ de las granjas de cuyes criaban, además, aves de corral. Se evaluaron cuyes machos y hembras, que se encontraban en etapas de recría y reproducción (198 animales de recría y 101 reproductores).

\section{Evaluación Clínica}

Los animales del estudio fueron seleccionados al azar. Se obtuvo información de las variables: etapa productiva (recría/reproductores) y sexo. Los cuyes fueron examinados para determinar la presencia de ectoparásitos y lesiones.
Recuperación de ectoparásitos (piojos y ácaros)

- Técnica del raspado de piel: Se realizó solo en los cuyes que presentaron lesiones dérmicas en cabeza y cuerpo (alopecia, queratinización o descamación). Se les hizo un raspado profundo de los bordes del área lesionada hasta evidenciar un leve sangrado. La muestra fue colocada en placas Petri $(5 \times 3 \mathrm{~cm})$ para su posterior procesamiento (Wall y Shearer, 2001; Radostits et al., 2002).

- Técnica de la cinta adhesiva transparente. En cuatro puntos del cuerpo (cabeza, región dorsal, región lumbosacra y vientre) se realizaron pequeñas presiones con cinta adhesiva transparente sobre la superficie corporal seleccionada. Luego, la cinta adhesiva fue colocada sobre una lámina portaobjeto para la observación microscópica en el laboratorio (Wall y Shearer, 2001; Radostits et al., 2002).

- Técnica de tricograma: En los cuatro puntos del cuerpo indicados previamente y con ayuda de una pinza Mayo se extrajo una muestra de pelos (aprox. 10 pelos/punto) de todos los animales. Las muestras de cada cuy se colocaron en una sola bolsa ziploc con alcohol al 80\% (Radostits et al., 2002).

- Técnica del peine fino. Se espolvorea sobre el cuerpo del cuy un polvo fino a base de propuxur para permitir el desprendimiento de los ectoparásitos. Luego de cinco minutos, se coloca el animal en una caja forrada con papel Kraft y se realiza el cepillado de toda la superficie corporal en dirección del pelaje y en forma contraria a este durante tres minutos. Se dobla el papel Kraf conteniendo el pelaje desprendido juntamente con los ectoparásitos y se le sella con cinta adhesiva (Wall y Shearer, 2001; Radostits et al., 2002).

\section{Procesamiento Parasitológico}

Se realizó un procesamiento específico en el laboratorio para cada técnica de recuperación: 
- Raspado de piel: Las muestras fueron vertidas en placas Petri con agua destilada. Bajo el estereoscopio, las estructuras compatibles con ectoparásitos en la superficie del agua fueron colectadas. Así mismo, con una pipeta Pasteur se obtuvo pequeñas cantidades de sedimento para su observación en el microscopio (10X y 40X).

- Cinta adhesiva: Las láminas fueron observadas directamente al microscopio, con adición de $\mathrm{KOH}$ al $10 \%$ a las muestras que requerían mejor visualización.

- Tricograma: La muestra fue dividida en dos porciones iguales. La primera fue observada directamente al microscopio con unas gotas de glicerina. La segunda porción fue colocada en una placa Petri y visualizada en el estereoscopio. Los especímenes encontrados fueron recolectados con pinzas entomológicas y puestos en viales con alcohol al $70 \%$.

- Peine fino: El material colectado fue vertido en 4-5 placas Petri con agua destilada y se realizaron varios lavados $(\sim 3)$ hasta eliminar las partículas del fármaco. Las muestras se observaron bajo el estereoscopio, según indicado para el raspado de piel. Los especímenes encontrados fueron tomados con pinzas entomológicas y puestos en viales con alcohol al $70 \%$.

Adicionalmente, se realizó el conteo de piojos y ácaros hallados en cada técnica, para determinar la intensidad media y el rango de infestación de los animales. La identificación del género y especies de los ectoparásitos se hizo con base a claves taxonómicas (Al-Rabiai et al., 1983; Soulsby, 1987; Wall y Shearer, 2001; Serra-Freire y Pinto, 2006; Taylor et al., 2016).

\section{Montaje de Ectoparásitos}

Una misma técnica de montaje fue utilizada para piojos (Gliricola porcelli) y para dos especies de ácaros (Dermanyssus gallinae y Ornithonyssus bursa), debido a la similitud de la característica de dureza de sus cutículas dura. Los especímenes conservados en alcohol al 70\% fueron sometidos a tres lavados con agua destilada durante 30 minutos $\mathrm{c} / \mathrm{u}$. Posteriormente, las muestras se colocaron en solución de $\mathrm{KOH}$ al $10 \%$ para su aclaramiento por 48 a 72 horas. Luego se procedió a un nuevo lavado con agua destilada, para iniciar un proceso de deshidratación por una batería de alcoholes seriados $(80,90$ y $100 \%$ ) durante 10 minutos por cada cambio. A continuación, se pasó por una mezcla de xylol y etanol (50:50) durante 15 minutos, para pasar las muestras por dos cambios de xylol puro por 15 minutos c/u. Finalmente, se realizó el montaje con Entellán ${ }^{\circledR}$ (Martín, 1994).

\section{Análisis de Datos}

Se calculó la prevalencia de ectoparásitos mediante la determinación del número de muestras positivas de cada parásito involucrados. Los resultados se presentan en forma porcentual con sus respectivos intervalos de confianza al 95\% (Daniel, 2007). Así mismo, se determinó la intensidad media y el rango por especie de ectoparásito, según lo establecido por Bush et al. (1997).

Se usó la prueba de Chi cuadrado para analizar la posible asociación entre las variables en estudio (sexo y etapa productiva) con la presencia de ectoparásitos. Los datos fueron procesados con el paquete estadístico STATA ${ }^{\circledR}$, v. 12.0.

\section{Resultados}

Se encontró una prevalencia general de $67.0 \pm 5.3 \%$, encontrándose ácaros y piojos. No se encontró asociación significativa entre las variables sexo y edad con el ectoparasitismo (Cuadro 1). El principal grupo de ectoparásitos correspondió a los ácaros con $63 \%$ de prevalencia, siendo Ornithonyssus bursa el ácaro más común (53\%). Se recuperaron 5922 ectoparásitos en total y el número promedio por especie de ectoparásito identificado (IM) se muestra en el Cuadro 2. 
Cuadro 1. Prevalencia de ectoparasitismo y asociación con las variables sexo y etapa productiva en 299 cuyes (Cavia porcellus) de crianza familiar-comercial del distrito de Matahuasi, provincia de Concepción, Junín. 2017

\begin{tabular}{llccc}
\hline Variables & & $\begin{array}{c}\text { Cuyes } \\
(\mathrm{n})\end{array}$ & $\begin{array}{c}\text { Positivos } \\
(\%)\end{array}$ & Valor $\mathrm{p}$ \\
\hline Sexo & Macho & 127 & 71.6 & 0.108 \\
& Hembra & 172 & 62.8 & \\
Etapa & Recría & 198 & 70.2 & 0.061 \\
productiva & Reproductores & 101 & 59.4 & \\
\hline Total \pm IC & & 299 & $67 \pm 5.3$ & \\
\hline
\end{tabular}

Cuadro 2. Intensidad media (IM) y rango, según especie de ectoparásitos en cuyes (Cavia porcellus) infestados del distrito de Matahuasi, provincia de Concepción, Junín. 2017

\begin{tabular}{llcccc}
\hline \multirow{2}{*}{ Orden } & \multirow{2}{*}{ Ectoparásitos } & \multicolumn{2}{c}{ Frecuencia } & \multicolumn{2}{c}{ Carga } \\
\cline { 2 - 6 } & & $\mathrm{n}$ & $\%$ & $\mathrm{IM}$ & Rango \\
\hline Acariformes & Ornithonyssus bursa & 157 & 53 & 29.3 & $1-286$ \\
(Ácaros) & Chirodiscoides caviae & 46 & 15 & 6.6 & $1-131$ \\
& Dermanyssus gallinae & 22 & 7 & 19.3 & $1-341$ \\
\cline { 2 - 6 } & Total de ácaros (\%) & 188 & 63 & 28.05 & $1-341$ \\
\cline { 2 - 6 } & Gliricola porcelli & 35 & 12 & 18 & $1-345$ \\
\cline { 2 - 6 } $\begin{array}{l}\text { Phthiraptera } \\
\text { (Piojos) }\end{array}$ & Total de piojos (\%) & 35 & 12 & 18.5 & $1-345$ \\
\hline Total & 199 & 67 & 29.8 & $1-345$ \\
\hline
\end{tabular}

En el $49.8 \%$ de las infestaciones solo se observó una especie parasitaria; por lo general Ornithonyssus bursa (Figura 1). Dos especies estuvieron presentes simultáneamente en el $13.4 \%$ de los cuyes con ectoparásitos, siendo Ornithonyssus bursa y Chirodiscoides caviae la combinación más común. Solo el 3.3\% tenían tres o más ectoparásitos presentes (Cuadro 3).

En cuanto a las técnicas de diagnóstico, el peine fino $(54.0 \pm 5.7 \%)$ fue la más eficiente en detectar el mayor número de cuyes con ectoparásitos, seguido de la cinta adhesiva $(41 \pm 5.57 \%)$ (Cuadro 4). No se evidenció la presencia de ácaros con la técnica de raspado profundo de piel. En todas las técnicas predominó la recolección de ácaros, principalmente de Ornithonyssus bursa.

\section{Discusión}

El presente estudio reporta una alta prevalencia de ectoparásitos $(67.0 \pm 5.3 \%)$ en cuyes de crianza familiar-comercial del dis- 
Cuadro 3. Tipo de parasitismo en cuyes (Cavia porcellus) de crianza familiarcomercial del distrito de Matahuasi, provincia de Concepción, Junín. 2017

\begin{tabular}{|c|c|c|c|c|c|}
\hline \multirow{2}{*}{$\begin{array}{l}\text { Tipos de } \\
\text { ectoparasitismo }\end{array}$} & \multicolumn{2}{|c|}{ Positivos } & \multirow{2}{*}{ Especies } & \multicolumn{2}{|c|}{ Especie } \\
\hline & $\mathrm{n}$ & $\%$ & & $\mathrm{n}$ & $\%$ \\
\hline \multirow[t]{4}{*}{ Monoparasitismo } & 149 & 49.8 & Ornithonyssus bursa & 114 & 38.1 \\
\hline & & & Chirodiscoides caviae & 18 & 6 \\
\hline & & & Gliricola porcelli & 10 & 3.3 \\
\hline & & & Dermanyssus gallinae & 7 & 2.3 \\
\hline \multirow[t]{6}{*}{ Biparasitismo } & 40 & 13.4 & O. bursa + C. caviae & 19 & 6.4 \\
\hline & & & O. bursa + G. porcelli & 10 & 3.3 \\
\hline & & & D. gallinae + O. bursa & 5 & 1.7 \\
\hline & & & D. gallinae + G. porcelli & 3 & 1 \\
\hline & & & C. caviae + G. porcelli & 2 & 0.7 \\
\hline & & & D. gallinae + C. cavia & 1 & 0.3 \\
\hline \multirow[t]{4}{*}{ Poliparasitismo } & 10 & 3.3 & $\begin{array}{l}\text { D. gallinae }+O . \text { bursa }+G . \\
\text { porcelli }\end{array}$ & 4 & 1.3 \\
\hline & & & $\begin{array}{l}\text { O. bursa }+ \text { C. caviae }+G \text {. } \\
\text { porcelli }\end{array}$ & 4 & 1.3 \\
\hline & & & $\begin{array}{l}\text { D. gallinae }+C . \text { caviae }+G \text {. } \\
\text { porcelli }\end{array}$ & 1 & 0.3 \\
\hline & & & $\begin{array}{l}\text { D. gallinae }+O \text {. bursa }+C \text {. } \\
\text { caviae }+G . \text { porcelli }\end{array}$ & 1 & 0.3 \\
\hline
\end{tabular}

Cuadro 4. Especies parasitarias identificadas con las técnicas de tricograma, cinta adhesiva y peinado fino en cuyes (Cavia porcellus) de crianza familiarcomercial del distrito de Matahuasi, provincia de Concepción, Junín. 2017

\begin{tabular}{llcccccc}
\hline & \multirow{2}{*}{ Especies } & \multicolumn{2}{c}{ Tricograma } & \multicolumn{2}{c}{ Cinta adhesiva } & \multicolumn{2}{c}{ Peine fino } \\
\cline { 2 - 8 } & $\mathrm{n}$ & $\%$ & $\mathrm{n}$ & $\%$ & $\mathrm{n}$ & $\%$ \\
\hline Acariformes & $\begin{array}{l}\text { Ornithonyssus } \\
\text { bursa }\end{array}$ & 67 & 22.4 & 87 & 29.1 & 131 & 43.8 \\
& $\begin{array}{l}\text { Chirodiscoides } \\
\text { caviae }\end{array}$ & 16 & 5.4 & 11 & 3.7 & 9 & 3.0 \\
$\begin{array}{l}\text { Dermanyssus } \\
\text { gallinae }\end{array}$ & 2 & 0.7 & 8 & 2.7 & 17 & 5.7 \\
Phthiraptera & Gliricola porcelli & 8 & 2.7 & 7 & 2.3 & 32 & 10.7 \\
\hline Total \pm IC & & 90 & $30 \pm 5.2$ & 121 & $41 \pm 5.6$ & 162 & $54 \pm 5.7$ \\
\hline
\end{tabular}




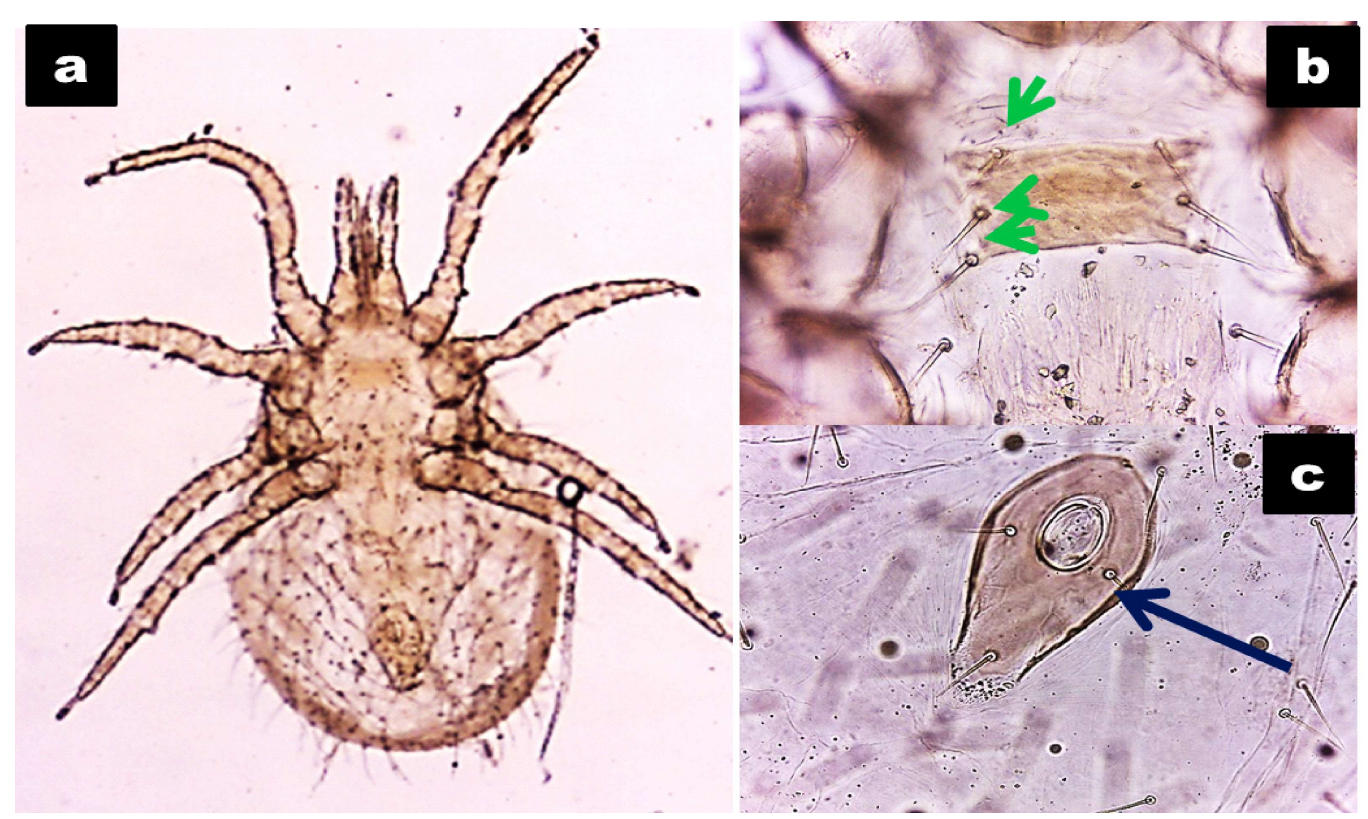

Figura 1. Características morfológicas de Ornithonyssus bursa. a. Espécimen observado en microscopio (10X y 40X). b. Placa esternal, nótese su forma y de los tres pares de setas (flecha verde). c. Placa anal (flecha azul)

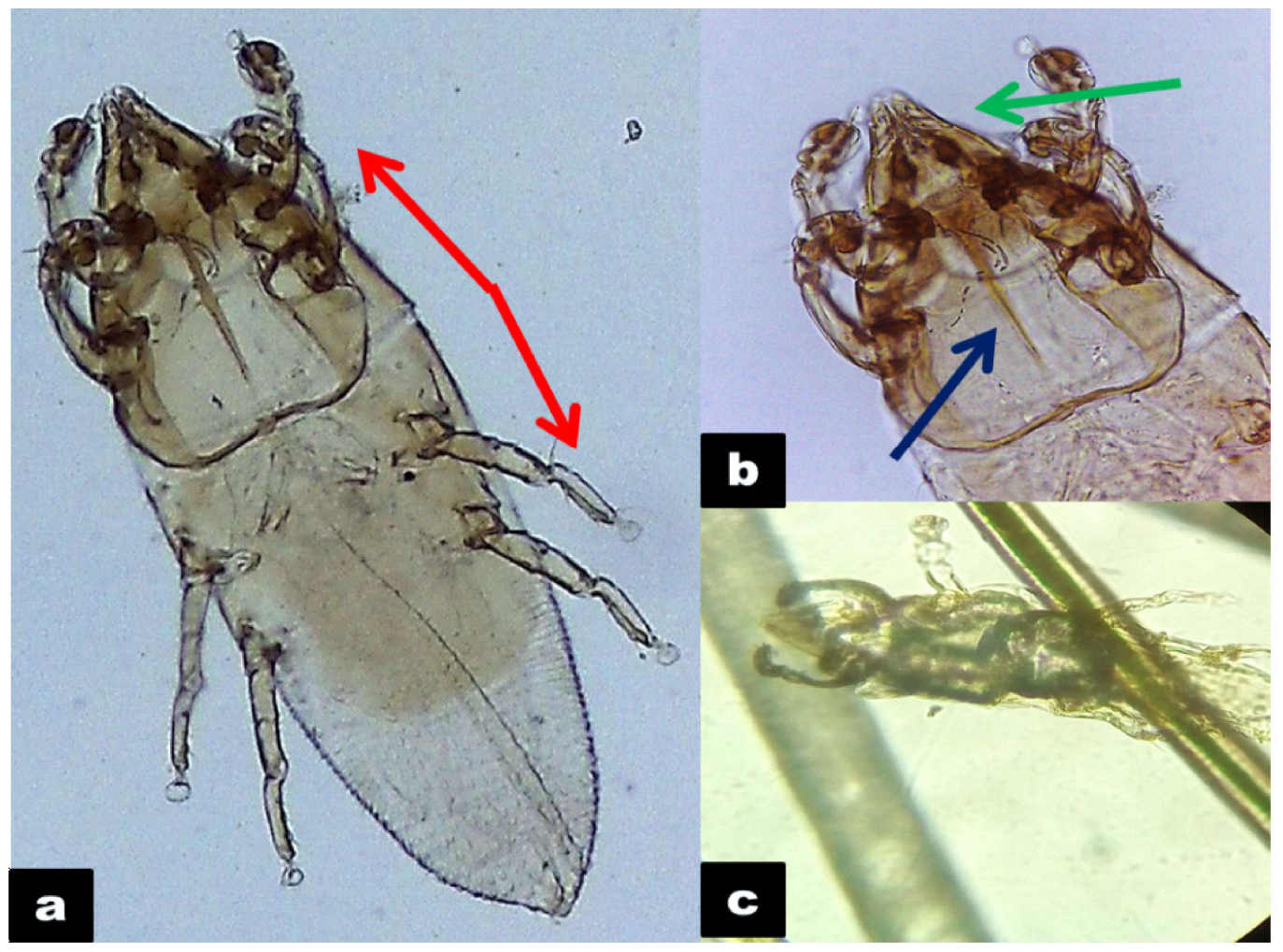

Figura 2. Características morfológicas de Chirodiscoides caviae. a. Vista ventral, nótese que los dos primeros pares de patas se encuentran más desarrollados que los posteriores (flecha roja). b. Gnotosoma (flecha verde) y Placa esternal estriada (flecha azul). c. Espécimen observado en microscopio (10X) 


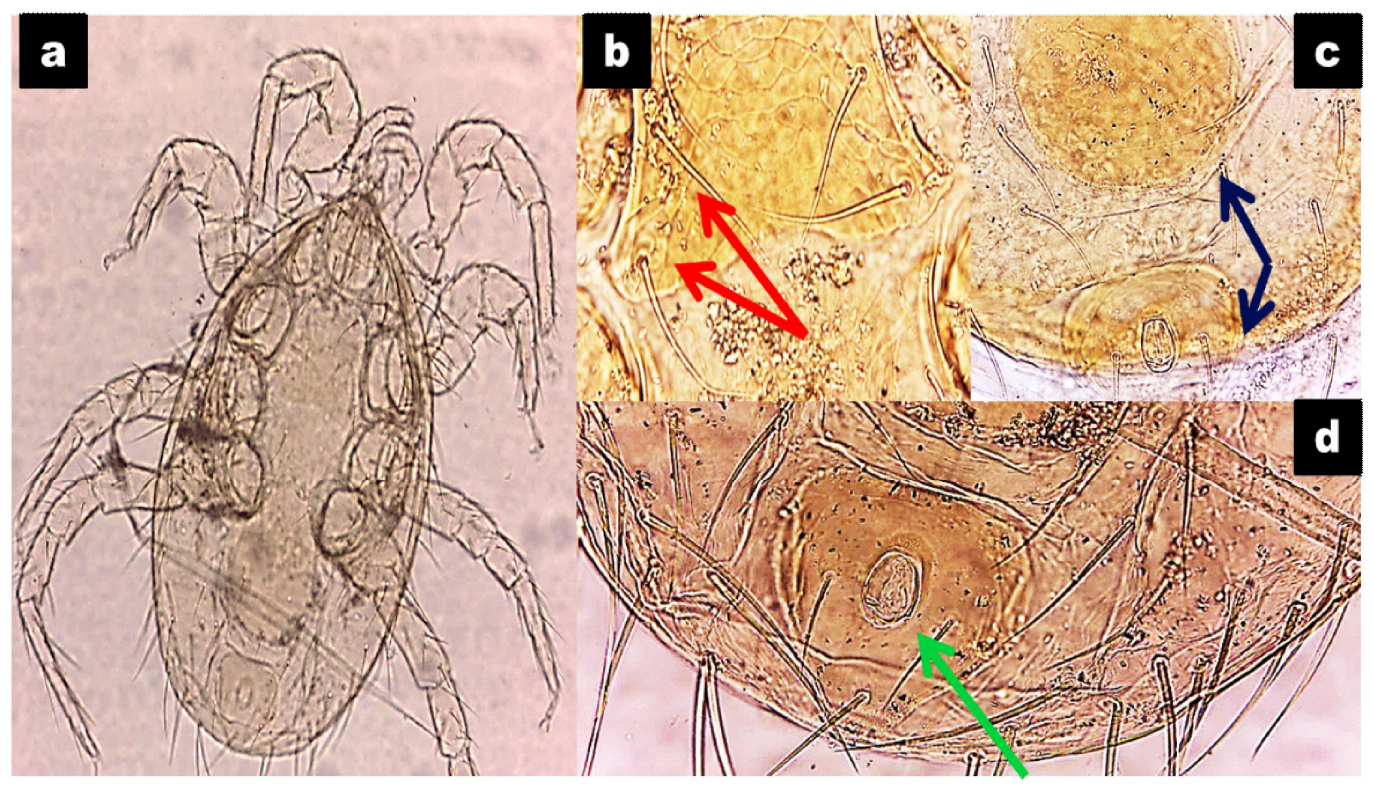

Figura 3. Características morfológicas de Dermanyssus gallinae. a. Espécimen observado en microscopio (10X y 40X). b. Placa esternal, nótese los dos pares de setas (flecha roja). c. Extremo posterior de la placa ventrogenital, nótese que es del mismo tamaño que la placa anal (flecha azul). d. Placa anal (flecha verde)

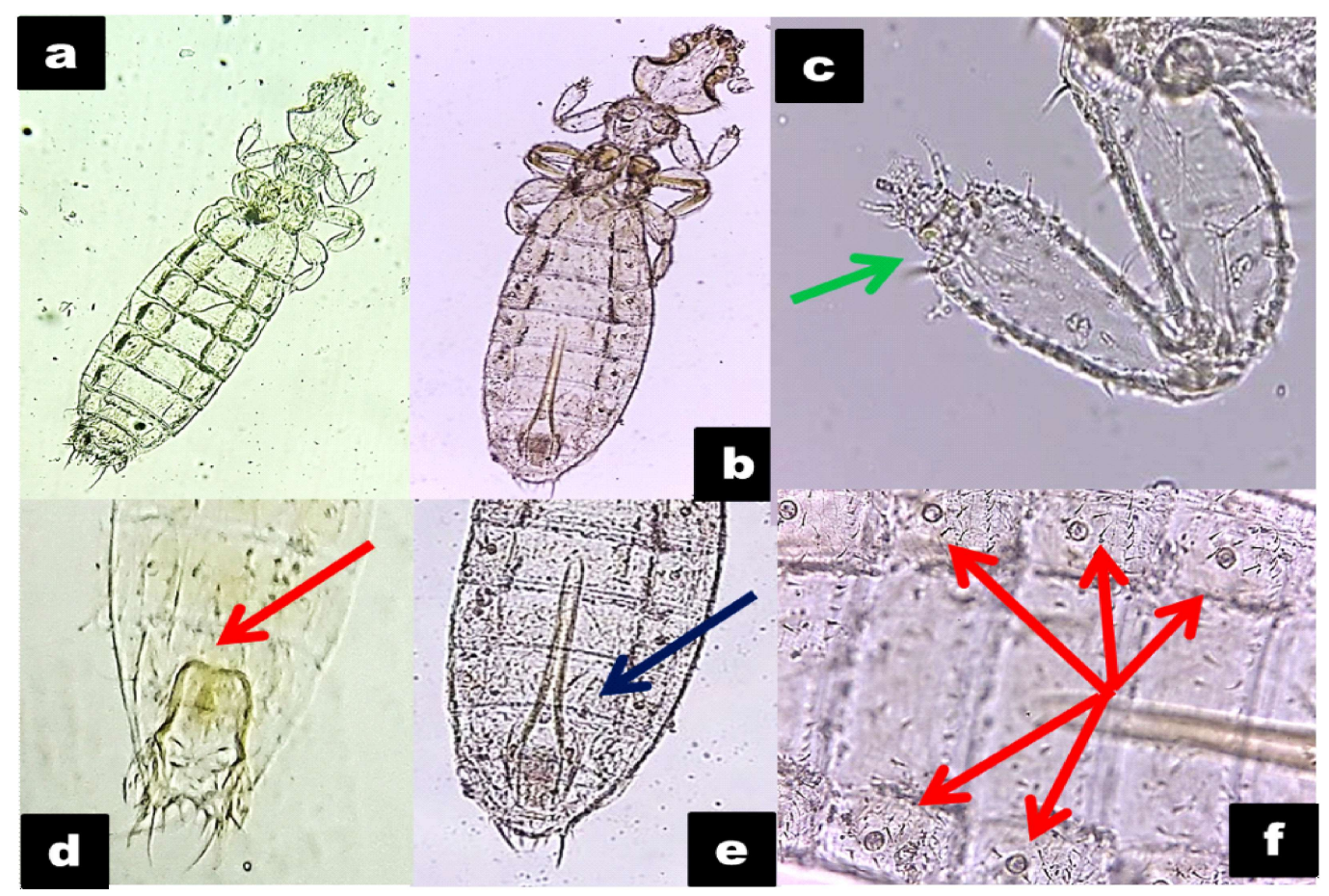

Figura 4. Características morfológicas de Gliricola porcelli. a y b. Especímenes observados en microscopio (10X y 40X). c. Tarso, obsérvese la falta de uña tarsal (flecha verde). d. Aparato reproductor de la hembra. e. Aparato reproductor del macho, mostrando el Aedeagus (flecha azul). f. Estigmas respiratorios (flechas rojas) 
trito de Matahuasi, Junín, Perú. El porcentaje observado es similar a los reportados por Chambilla (2012) de 59.1\% y Gordillo (2015) de $75.5 \%$ en Tacna y Arequipa, respectivamente. Sin embargo, Dittmar (2001) y Aguilar et al. (2011) señalan frecuencias superiores al $90 \%$ en diversas zonas de la costa, sierra y selva del país. Estas diferencias se deben principalmente a la evaluación de cuyes de crianza familiar, donde la infraestructura y el control sanitario es deficiente.

No se encontró asociación significativa entre la presencia de ectoparásitos y las variables sexo y etapa productiva; es decir, estas variables no constituyeron factores influyentes para la presentación del ectoparasitismo en cuyes, posiblemente debido a que todos estuvieron sujetos a similares condiciones de manejo y exposición del agente.

Se llegó a recuperar 5922 ectoparásitos, de los cuales 5274 fueron ácaros y 648 fueron piojos. El grado de infestación de Chirodiscoides caviae (Figura 2), Ornithonyssus. bursa (Figura 1) y Dermanyssus gallinae (Figura 3), fue probablemente leve, dada que la presencia media de más de 1500 ácaros, principalmente $D$. gallinae, lo cual considerado como infestación media (Chauca, 1997).

Se encontró mayor frecuencia de ácaros $(63 \%)$ que de piojos (12\%), no hallándose la presencia de pulgas. Diversos autores coinciden en señalar que los ácaros superficiales son los ectoparásitos predominantes en cuyes (Dittmar, 2001; Aguilar et al., 2011; Chambilla, 2012; Gordillo, 2015), a diferencia de los señalado por Cadenillas (2017), quien encontró una mayor frecuencia de piojos en el norte del país. Entre los ácaros identificados, prevaleció Ornithonyssus bursa (53\%), considerado un parásito hematófago común en aves (Wall y Shearer, 2001). Son escasos los estudios que logran identificar la especie del ácaro hallado a través del aclaramiento y observación de estructuras morfológicas, ya que Ornithonyssus y Dermanyssus son macroscópicamente similares, de allí la importancia del uso de claves taxonómica para su identificación; entre ellas estructuras como el escudo dorsal, placa esternal, ventrogenital y anal, así como la disposición del ano (Dittmar, 2001; Robles et al., 2014; Gordillo, 2015; Cadenillas, 2017). En algunos estudios se señala el género Ornithonyssus o se alude erróneamente al denominado ácaro rojo o chuchuy, que corresponde a otro ácaro «Dermanyssus» (Chambilla, 2002; Aguilar et al., 2011).

Estudios realizados en el país han identificado otras especies de Ornithonyssus, tales como $O$. sylviarum por Flores et al. (2010) en Ancash, quienes mencionan la transmisión en cuyes se debería por la presencia de aves en los galpones; en tanto que Dittmar (2001) en Huancayo encontró Ornithonyssus bacoti, cuya fuente de transmisión es básicamente por roedores (Rattus spp).

El perjuicio de $O$. bursa es variable según la especie hospedadora y su efecto puede ser no medible hasta conllevar una pérdida significativa de sangre, por sus características hematófagas y cursar con mortalidad en infestaciones severas (Szép y Møller, 2000). Este ácaro es causante de pérdidas de peso y anemia en varias especies de aves, incluyendo el gorrión (Passer domesticus) (Weddle, 2000) y golondrina (Hirundo rustica) (Møller, 1993; Morishita y Schaul, 2007). Sin embargo, las consecuencias patológicas en el cuy no han sido determinadas.

Otro ácaro, macroscópicamente similar al Ornithonyssus, pero con diferencias biológicas, lo constituye Dermanyssus gallinae, hallado en baja frecuencia en el presente estudio (7\%). Se le considera una plaga importante de aves de corral en todo el mundo (Hoglund et al., 1995) y ha quedado demostrado su papel como vector de la salmonelosis en aves ponedoras (Dhooria, 2016), por lo cual no se descartaría similar impacto en el sector cavícola, donde brotes causados por esta enfermedad producen alta mortalidad (Morales, 2017). 
La menor frecuencia de Dermanyssus gallinae en comparación a $O$. bursa puede explicarse por el tiempo de permanencia del ácaro sobre el hospedero. Parte del ciclo de vida de $D$. gallinae lo desarrolla en el ambiente y solo bajo condiciones de baja iluminación, generalmente por las noches, busca al cuy para alimentarse. Por el contrario, $O$. bursa permanece la mayor parte del tiempo sobre el hospedero, desarrollando todo su ciclo de vida en el cuy (McClain et al., 2009; Lareschi et al., 2017). Por otro lado, en el $26 \%$ de las granjas cavícolas se criaba, además, aves como patos, gallinas y loros, los cuales constituirían una fuente de infestación de $O$. bursa y D. gallinae.

Chirodiscoides caviae (Figura 2) presentó una baja prevalencia ( $15 \%)$, difiriendo del estudio de Robles et al. (2014), quienes hallaron prevalencias de 57\% en Oxapampa asociado a las condiciones geográficas, climáticas y al mayor brillo solar, característico de ceja de selva, lo que favorecería una mayor multiplicación y supervivencia de los ácaros (Jofré et al., 2009); a diferencia del distrito de Matahuasi que se encuentra en un valle interandino y de bajas temperaturas.

Chirodiscoides caviae se caracteriza por encontrarse generalmente adherido al tallo del pelo y es relativamente inofensivo, rara vez produciría problemas de caspa y eritema. Sin embargo, en infestaciones severas puede llevar a generar auto traumas y dermatitis ulcerativa (Paterson, 2006). d'Ovidio y Santoro (2014) señalaron 33\% de frecuencia en tiendas de mascotas y en animales criados como mascotas. A pesar de ser considerado específico de hospedero, algunos autores señalan su presencia en ratas de laboratorio (Harikrishnan et al., 2009).

Con relación al orden Phthiraptera, en el país se han descrito la presencia de tres especies (Gliricola porcelli, Gyropus ovalis y Trimenopon hispidum) en Cavia porcellus (Dittmar, 2001; Dittmar et al., 2003; Flores et al., 2010; Robles et al.; 2014; Cadenillas,
2017). Sin embargo, en el presente trabajo solo se halló al piojo Gliricola porcelli (12\%; Figura 4). Diversos estudios coinciden en señalarlo como especie dominante, además de ser considerado benigno, sin estar asociado a problemas de salud pública (Wall y Shearer, 2001; Mullen y Durden, 2002).

Si bien no se encontraron pulgas (orden Siphonaptera), varios autores han señalado su presencia (Pulex irritans, Tiamastus cavicola, Ctenocephalis felis, Xenopsylla cheopis, Echidnophaga gallinacea) en cuyes (Dittmar, 2001; Dittmar et al., 2003; Gordillo, 2015; Cadenillas, 2017). La diversidad y variedad de pulgas en cuyes suele estar asociada a la presencia de animales en las granjas como perros, gatos, conejos, aves, roedores, etc. Estos animales se encontraban en las granjas del estudio; sin embargo, la temperatura $\left(16^{\circ} \mathrm{C}\right)$ y humedad $(52-72 \%)$ de la zona fueron desfavorables para el desarrollo de dichos ectoparásitos (Soulsby, 1987; Quiroz, 2005; Weather Sspark, 2018).

En el presente estudio se encontró una baja asociación parasitaria en los cuyes evaluados, donde solo el 3.3\% presentaron tres o más especies de ectoparásitos, a diferencia de lo señalado por Dittmar et al. (2003), quienes encontraron casos de poli-infestación de hasta siete especies, incluyendo pulgas, piojos y ácaros, favorecido principalmente por una alta densidad de la población, inadecuada alimentación, enfermedades crónicas y animales relativamente viejos.

La mayoría de los cuyes no evidenciaron lesiones visibles en la piel (79.9\%), posiblemente debido a las cargas de infestaciones relativamente bajas. Así también, no se evidenciaron ácaros que pudieran afectar el estrato subdérmico de la piel. Los cuyes pueden ser afectados por ácaros estrictamente específicos como Demodex caviae y Trixacarus cavie, asociado en el primer caso a prurito recurrente, así como a problemas de inmunodeficiencia y el segundo caso al deficiente manejo (Schönfelder et al., 2010). 


\section{Conclusiones}

- La prevalencia de ectoparásitos en cuyes (Cavia porcellus) de crianza familiar-comercial del distrito de Matahuasi, provincia de Concepción, Junín, fue de $67.0 \pm 5.3 \%$

- Los ectoparásitos encontrados fueron ácaros: Ornithonyssus bursa 53\%, Chirodiscoides caviae 15\%, Dermanyssus gallinae 7\%, y el piojo Gliricola porcelli $12 \%$.

- Las asociaciones parasitarias fueron: monoparasitismo (49.8\%), donde Ornithonyssus bursa (38.1\%) fue el más frecuente; biparasitismo (13.4\%) con Ornithonyssus bursa más Chirodiscoides caviae (6.4\%) y poliparasitismo (3.3\%).

- No se encontró asociación significativa entre las variables sexo y edad con el ectoparasitismo.

\section{Literatura Citada}

1. Aguilar G, Bustamante J, Bazán V, Falcón N. 2011. Diagnóstico situacional de la crianza de cuyes en una zona de Cajamarca. Rev Inv Vet Perú 22: 9-14. doi: 10.15381/rivep.v22i1.113

2. Al-Rabiai S, Wagner J, Enns W, Farrar P.1983. A redescription of Chirodiscoides caviae Hirst (Acari: Atopome-lidae), with differentiating characteristics of male and female adult and nymphal stages. J Kansas Entomol Soc 56: 483-495.

3. Bush A, Lafferty K, Lotz J, Shostak A. 1997. Parasitology meets ecology on its own terms: Margolis et al. Revisited. J Parasitol 83: 575-583. doi: 10.2307/ 3284227

4. Cadenillas A. 2017. Prevalencia de ectoparásitos en cuyes (Cavia porcellus) de la ciudad de Ferreñafe - Departamento de Lambayeque. Tesis de Médico Veterinario. Lambayeque: Univ. Pedro Ruiz Gallo. 55 p.
5. Chambilla E. 2012. Diagnóstico de la producción de cuyes (Cavia porcellus) en la provincia de Tacna-2012. Tesis de Médico Veterinario y Zootecnista. Tacna: Univ. Jorge Basadre Grohmann. $130 \mathrm{p}$.

6. Chauca L. 1997. Producción de cuyes (Cavia porcellus). Estudio FAO producción y sanidad animal 138. Roma: FAO. $80 \mathrm{p}$.

7. d'Ovidio D, Santoro D. 2014. Prevalence of fur mites (Chirodiscoides caviae) in pet guinea pigs (Cavia porcellus) in southern Italy. Vet Dermatol 25: 135-137. doi: 10.1111/ vde. 12110

8. Daniel W. 2007. Bioestadística: base para el análisis de las ciencias de la salud. $4^{\circ}$ ed. México DF: Limusa. 924 p.

9. Dhooria M. 2016. Medical and veterinary acarology. In: Dhooria M (ed). Fundamentals of applied acarology. Singapur: Springer. p 425-439.

10. Dittmar K, Ribbeck R, Daugschies A. 2003. Vorkommen und verbreitung von ektoparasiten bei meerschweinchen $(\mathrm{Ca}$ via spp.) in Peru, Südamerika. Berl Munch Tierarztl 116: 102-107.

11. Dittmar K. 2001. Untersuchungen zum vorkommen von ektoparasiten bei domestizierten und wildlebenden meerschweinchen (Cavia spp) sowie an präinkaischen meerschweinchenmumien in Peru, Südamerika. PhD Thesis. Leipzig: Universität Leipzig. $156 \mathrm{p}$.

12. Flores S, Chávez A, Morales S. 2010. Ectoparásitos en cobayos (Cavia porcellus) del distrito de San Marcos Huaraz. En: XXII Congreso Panamericano de Ciencias Veterinarias PANVET. Lima.

13. Florián A. 1999. Pérdidas de producción debido a enfermedades parasitarias. En: V Congreso Latinoamericano de Cuyicultura. Puerto Ayacucho, Venezuela:

14. Gordillo R. 2015. Prevalencia de ectoparásitos en cuyes (Cavia porcellus) en el distrito de Santa Isabel de Siguas provincia de Arequipa. Tesis de Médico Veterinario y Zootecnista. Arequipa, Perú: Univ. Católica de Santa María. 87 p. 
15. Harikrishnan V, Ranaraj V, Fernan$\operatorname{dez} A$. 2009. Incidence of Chiro-discoides caviae in laboratory-rats screening: identification and treatment. Scand J Lab Anim Sci 36: 147-153.

16. Hoglund J, Nordenfor H, Uggla, A. 1995. Prevalence of the poultry red mite, Dermanyssus gallinae, in different types of production systems for egg layers in Sweden. Poultry Sci 74: 17931798. doi: 10.3382/ps.0741793

17. Jofré L, Noemí L, Neira P, Saavedra T, Díaz C. 2009. Acarosis y zoonosis relacionadas. Rev Chil Infectol 26: 248257. doi: 10.4067/S0716-10182009000400008

18. Lareschi M, Cicuttin G, De Salvo M, Ibañez L, Montalti D. 2017. The tropical fowl mite Ornithonyssus bursa (Acari: Mesostigmata: Macronyssidae) parasitizing the European starling Sturnus vulgaris (Aves: Passeriformes: Sturnidae), an invasive bird in central Argentina. An approach to the bacterial fauna of this mite. Rev Mex Biodivers 88: 454-458. doi: 10.1016/j.rmb.2017.03.022

19. Martín M. 1994. Manual de recolección y preparación de ectoparásitos (Malófagos, Anopluros, Sifonapteros y ácaros). Vol 3. Madrid: Consejo superior de investigaciones científicas. $80 \mathrm{p}$.

20. McClain D, Dana A, Goldenberg G. 2009. Mite infestations. Dermatol Therapy 22: 327-346. doi: $10.1111 / \mathrm{j} .1529$ 8019.2009.01245.x

21. Moller A. 1993. Ectoparasites increase the cost of reproduction in their hosts. $\mathrm{J}$ AnimEcol 62: 309-322. doi: 10.2307/5362

22. Morales S. 2017. Patógenos bacterianos y parasitarios más frecuentes de crianza familiar - comercial en tres distritos de la provincia de Bolognesi, Departamento de Ancash en época de seca. Tesis de Maestría. Lima: Univ. Nacional Mayor de San Marcos. 79 p.

23. Morishita T, Schaul J. 2007. Parasites of birds. In: Baker D (ed). Flynn's parasites of laboratory animals. $2^{\text {nd }} \mathrm{ed}$. Iowa: Blackwell Publishing. p 217-230.
24. Mullen G, Durden L. 2002. Medical and veterinary entomology. USA: Elsevier Science. 597 p.

25. Paterson S. 2006. Skin diseases and treatment of guinea pigs. In: Paterson $\mathrm{S}$ (ed). Skin diseases of exotic pets. Oxford: Blackwell Science. p 238-240.

26. Quiroz H. 2005. Parasitología y enfermedades parasitarias de animales domésticos. México DF: Limusa. $876 \mathrm{p}$.

27. Radostits O, Mayhew I, Houston D. 2002. Examen y diagnóstico clínico en veterinaria. Madrid: Harcourt. $771 \mathrm{p}$.

28. Robles K, Pinedo R, Morales S, Chávez, A. 2014. Parasitosis externa en cuyes (Cavia porcellus) de crianza familiar-comercial en las épocas de lluvia y seca en Oxapampa, Perú. Rev Inv Vet Perú 25: 51-57. doi: 10.15381/rivep.v25i1 8467

29. Schönfelder J, Henneveld K, Schönfelder A, Hein A, Müller R. 2010. Case report. Concurrent infestation of Demodex caviae and Chirodiscoides caviae in a guinea pig. Tierärztl Prax 38: 28-30. doi: 10.1055/s-00381622826

30. Serra-Freire N, Pinto R. 2006. Entomologia y acarología na Medicina Veterinária. Rio de Janeiro: Livros. 199 p.

31. Soulsby E. 1987. Parasitología y enfermedades parasitarias en los animales domésticos. $7^{\mathrm{a}}$ ed. México DF: Oficina Sanitaria Panamericana. $820 \mathrm{p}$.

32. Szép T, Moller A. 2000. Exposure to ectoparasites increases within-brood variability in size and body mass in the sand martin. Oecologia 125: 201-207. doi: $10.1007 / \mathrm{s} 004420000447$

33. Taylor M, Coop R, Wall R. 2016. Veterinary parasitology. $4^{\text {th }}$ ed. Oxford: Wiley Blackwell. $1024 \mathrm{p}$.

34. Valim M, Amorin A, Serra-Freire N. 2004. Parasitismo por Acari e Phthiraptera em cobaios Cavia porcellus (Linnaeus, 1758) de ambientes rural e urbano nos municipios de Silva Jardim e Duque de Caxias, Rio de Janeiro, Brasil. Braz J Vet Res Anim Sci 41: 240-246. doi: 10.1590/S1413-95962004000-400004 
35. Wall R, Shearer D. 2001. Veterinary ectoparasites: Biology, pathology and control. $2^{\text {nd }}$ ed. Oxford: Blackwell Science. $262 \mathrm{p}$.

36. Weather Spark. 2018. Clima promedio de Matahuasi Perú. [Internet]. Disponible en: https://es.weatherspark.com/y/
22329/Clima-promedio-en-MatahuasiPer\%C 3\%B A-durante-todo-el$\mathrm{a} \% \mathrm{C} 3 \% \mathrm{~B} 1 \mathrm{o}$

37. Weddle CB. 2000. Effects of ectoparasites on nestling body mass in the house sparrow. Condor 102: 684-687. doi: 10.1650/0010-5422(2000)102 